\title{
Spectral Characterization of the AisaOWL
}

\author{
Laura Harris, Mark Warren, Mike Grant, and Gary M Llewellyn
}

\begin{abstract}
The AisaOWL is a recent-to-market thermal hyperspectral instrument. As such, there is little information about the sensor performance in the literature. The sensor covers the 7.6-12.6 $\mu \mathrm{m}$ part of the long-wave infrared region with 102 continuous bands, and is capable of imaging in low-light conditions. This paper presents an independent characterization of the AisaOWL sensor, examining the spectral accuracy of black body measurements at different temperatures and validating manufacturer recommendations for warm-up, integration, and calibration times. This analysis is essential for establishing high quality operational procedures and in giving confidence to users of the data. In this paper, the sensor has been found to have a maximum error of $2{ }^{\circ} \mathrm{C}$ in absolute temperature measurement, and provides spectra most accurate in the 8-9 $\mu \mathrm{m}$ region. The recommended warm-up time of $15 \mathrm{~min}$ has been confirmed, with a $1 \%$ increase in error identified for data collected only 7 min after switch on. The optimal integration time of $1.18 \mathrm{~ms}$ has been validated and an exponential decrease in performance observed outside the $0.85-1.2 \mathrm{~ms}$ range. The detector used by the sensor is shown to have stability issues and this has been examined by comparing black body data processed with different calibration data. While the detector is operating in a stable regime compatible with the calibration, these black body readings stay within $5 \%$ across the central bands, approaching $10 \%$ below $8 \mu \mathrm{m}$ and just exceeding $20 \%$ above $11 \mu \mathrm{m}$.
\end{abstract}

Index Terms-AisaOWL, calibration, hyperspectral, thermal.

\section{INTRODUCTION}

$\mathbf{R}$ EMOTE sensing is an established technique that can be used to acquire information over large, potentially hazardous regions. Earth observation remote sensing is typically performed from airborne or satellite systems. Satellite data offer regular coverage over large areas without any airspace restrictions but generally at lower spatial and temporal resolution, whereas aircraft offer a solution to demands for rapid access to higher resolution data. Both platforms complement one another, with airborne data often being used to calibrate and validate satellite data [1].

Typical applications, which use airborne remote sensing data, include vegetation characterization [2], forestry mapping [3], species classification [4], water toxicity [5], and geological studies [6].

Manuscript received September 2, 2016; revised October 25, 2016; accepted January 5, 2017. Date of publication February 24, 2017; date of current version March 17, 2017. This work was supported in part by the Natural Environment Research Council under the Natural Environment Research Council Airborne Research Facility and in part by the Icelandic Research Fund through the Grant of Excellence Project Environmental Mapping and Monitoring of Iceland by Remote Sensing.

L. Harris, M. Warren, and M. Grant are with the Plymouth Marine Laboratory, Plymouth, PL1 3DH (PML), U.K. (e-mail: lah@pml.ac.uk; mark1@pml.ac.uk; mggr@pml.ac.uk).

G. Llewellyn is with British Antarctic Survey, Cambridge, CB3 OET (BAS), U.K. (e-mail: gaew@nerc.ac.uk).

Color versions of one or more of the figures in this paper are available online at http://ieeexplore.iee.org.

Digital Object Identifier 10.1109/TGRS.2017.2653241
Thermal remote sensing can provide measurements of surface energy fluxes and temperatures, so has applications in the analysis of landscape ecological processes [7], soil content analysis [8], and even in the detection of disturbed earth for mine clearance purposes [9]. Thermal hyperspectral remote sensing is also particularly useful for mineral mapping, as minerals have distinct differences in the longwave infrared (LWIR) spectral reflectance, unlike the visible and near infrared (VNIR), short-wave infrared (SWIR), and midwave infrared regions [10].

Recent improvements in sensor design have led to viable airborne and satellite hyperspectral thermal instruments, including the NASA Hyperspectral Thermal Emission Spectrometer [11], Telops HYPER-CAM, the Itres Thermal Airborne Spectrographic Imager (TASI), and the Specim AisaOWL. Further information on each of these sensors can be found from manufacturer websites. The AisaOWL has been selected by the Natural Environment Research Council to provide an airborne environmental data service alongside the AisaFENIX, the equivalent VNIR-SWIR hyperspectral sensor, a Leica ALS50-II lidar, and a medium format digital camera.

This paper presents the results of an independent characterization of the Specim AisaOWL thermal hyperspectral instrument. This analysis is essential for establishing high quality operational procedures and in giving confidence to users of the data. This paper is organized as follows. Section II provides an overview of the AisaOWL sensor with key specifications and example data. Section III outlines the methodology of the calibration procedures followed. Section IV presents the results and discussion of the various calibration tests. Finally, the conclusions about the AisaOWL performance are summarized in Section VI.

\section{AisaOWL}

The AisaOWL is a thermal hyperspectral instrument sensitive to LWIR radiation, specifically $7.6-12.6 \mu \mathrm{m}$, with 102 continuous bands. Table I gives an overview of the sensor specification; further details are available from the manufacturer.

The AisaOWL has a mercury cadmium telluride detector array, which has an inherent randomly varying dark current for random pixels. This manifests in the raw data as a pixel switching between different intensity levels. These pixels are termed blinkers by the manufacturer. They do react as expected to different light intensities, apart from this switching behavior, so are not bad pixels. Blinking behavior occurs randomly, so pixels must be identified for each data acquisition. It is planned to characterize blinking pixel behavior in a dedicated study, so blinkers will not be further discussed in this report.

In measuring thermal emissions, the AisaOWL is capable of spectral imaging and material classification in low-light

0196-2892 @ 2017 IEEE. IEEE. Translations and content mining are permitted for academic research only. Personal use is also permitted, but republication/redistribution requires IEEE permission. See http://www.ieee.org/publications_standards/publications/rights/index.html for more information. 
TABLE I

SPECIFICATION OF THE Ais AOWL

\begin{tabular}{|c|c|}
\hline Measurement & Value \\
\hline Spectral bands & 102 \\
Spatial pixels & 384 \\
Measured FOV (total) & $24.20^{\circ}$ \\
Measured FOV (from centre) & $-11.79^{\circ}, 12.41^{\circ}$ \\
Paraxial FOV & $-11.80^{\circ} 12.39^{\circ}$ \\
Sensor IFOV & $0.063^{\circ}$ or $1.103 \mathrm{mrad}$ \\
Lens focal length & 42.886 \\
Optical axes position (central pixel) & 187.7 \\
Max distortion & $0.0755 \mathrm{~mm}$ or $0.0210^{\circ}$ or $0.171 \%$ \\
\hline
\end{tabular}

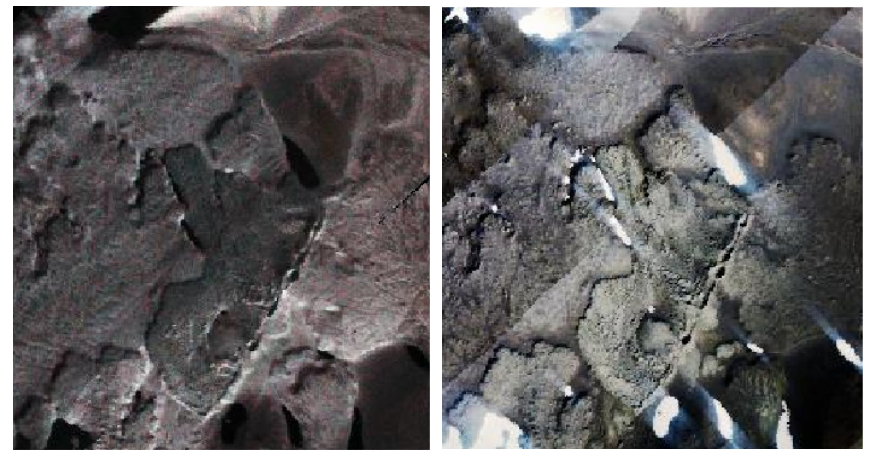

Fig. 1. At-sensor radiance data of Iceland's volcanic Hekla region. (Left) AisaOWL mosaic. (Right) True color AisaFENIX mosaic.

conditions, detection and classification of emitted gas, and surface temperature estimations given known emissivity [12]. Methods do exist to extract temperature and/or emissivity from thermal imagery without knowledge of the other, but assumptions must be made to reduce the number of unknown contributions to the at-sensor radiance. These are further complicated by atmospheric correction and surface multiple scattering [13]. A summary of various methods for extracting emissivity form thermal infrared images is presented in [14]. Combined with SWIR hyperspectral imagery, improved classification of minerals, particularly silicates, is possible [10].

The AisaOWL has already acquired high resolution thermal imagery in a range of challenging conditions, including the tropical rainforests of Malaysia and the frozen volcanic contrasts of Iceland. Fig. 1 shows AisaOWL data acquired over Iceland's volcanic Hekla region alongside true color imagery captured simultaneously with the AisaFENIX. The bright and dark patterns in the AisaOWL image may indicate temperature differences in the rocks, or different emissivity properties. The dark regions that correspond to the bright regions in the AisaFENIX data are snow.

During normal operation, the sensor is calibrated at the end of each acquisition using an integrated pair of black bodies, which are mechanically moved in front of the sensor lens. The two black bodies are at known temperatures, set by the operator such that they bound the expected temperature of the scene. Typically 1024 frames are acquired from both of the internal black bodies, which takes approximately $3 \mathrm{~min}$ when the sensor is functioning correctly. This is essential, because each time the sensor is turned on the detector response is

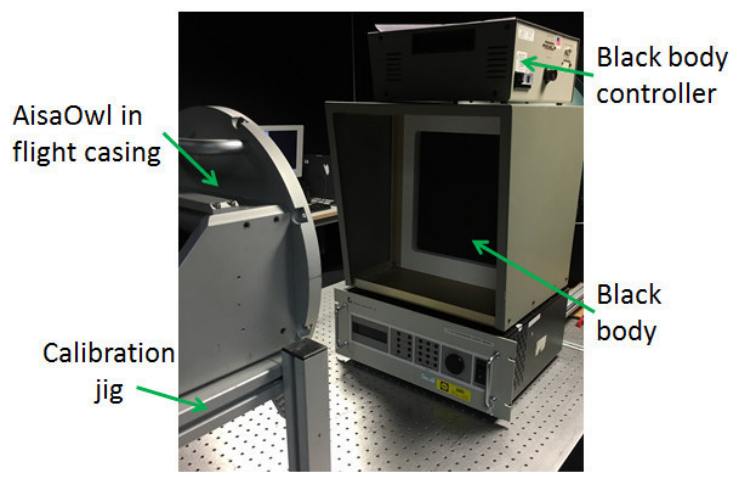

Fig. 2. Experimental setup for black body data acquisition.

different and changes over a short time period, so cannot be calibrated with a bench calibration. The manufacturer states that it is stable over a period of $30 \mathrm{~min}$, so should be calibrated at least every 30 -min interval.

\section{Methodology}

The AisaOWL is calibrated using internal black bodies for each acquisition, so unlike other hyperspectral instruments that detect light in the VNIR and SWIR, every time data are collected it is calibrated by different calibration gains. However, an external bench calibration provides an opportunity to evaluate performance and sensor characteristics.

During the bench calibration, the AisaOWL was secured to an optics table aligned with an Infrared Systems Development Corporation IR-160 tunable black body, as shown in Fig. 2. The temperature of the black body was verified with a forward looking infrared (FLIR). The sensor was positioned close enough to the black body so that each detector pixel was exposed only to the uniform radiant output. The temperature of the black body was varied from $40{ }^{\circ} \mathrm{C}$ to $100{ }^{\circ} \mathrm{C}$ in steps of $10{ }^{\circ} \mathrm{C}$. Additional measurements at $20{ }^{\circ} \mathrm{C}$ and $30{ }^{\circ} \mathrm{C}$ are provided from a later bench calibration.

Additional tests were conducted with this setup. Data were collected from the black body set to a temperature of $40{ }^{\circ} \mathrm{C}$ with an integration time varying between 0.18 and $2 \mathrm{~ms}$. Data were also collected prior to the recommended warm-up time of 15-30 min. Approximately $30 \mathrm{~s}$ of data were collected for each test at a frame rate of $100 \mathrm{frames} / \mathrm{s}$, corresponding to approximately 3000 scan lines.

Data were processed using version 2.2 of Specim's calibration software, using the default settings. These settings apply some spatial and spectral averaging to smooth the data and replace some pixels with nearest good neighbor data if they have been identified as bad or blinking. This paper focuses on the characterization of the entire detector array without examining any spatial pixel effects and uses an average spectrum across all spatial pixels and lines for each data set. Each data set was calibrated at the time of data acquisition using the internal black body sources, with some data being processed again with alternative calibration data to test the stability of the instrument calibration.

Processed averaged spectra were compared against spectra calculated using Planck's Law for black body radiation [see (1)] to calculate either a percentage difference 


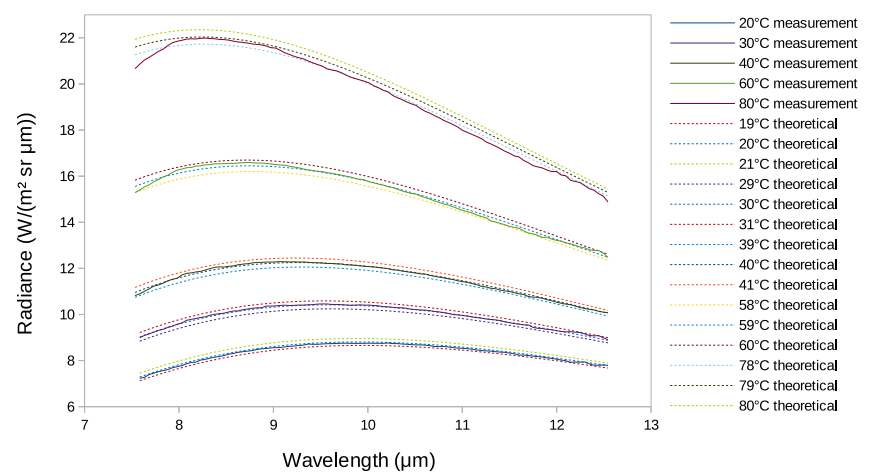

Fig. 3. Measured spectra of a $20{ }^{\circ} \mathrm{C}, 30{ }^{\circ} \mathrm{C}, 40{ }^{\circ} \mathrm{C}, 60^{\circ} \mathrm{C}$, and $80{ }^{\circ} \mathrm{C}$ black body alongside theoretical black body spectra.

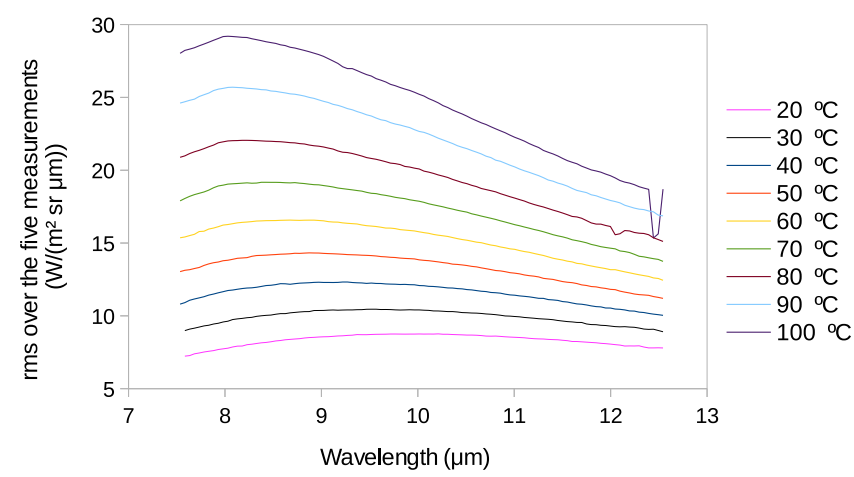

Fig. 4. RMS of each of the five measurements at the different temperatures.

or root-mean-squared (rms) error

$$
B_{\lambda}(T)=\frac{2 h c^{2}}{\lambda^{5}} \times \frac{1}{e^{h c / \lambda k_{B} T}-1}
$$

where $B_{\lambda}(T)$ is the radiant energy at wavelength $\lambda$ and temperature $T, h$ is Planck's constant, $c$ is the speed of light, and $k_{B}$ is the Boltzmann constant.

\section{RESUltS AND Discussion}

\section{A. Temperature Validation}

Fig. 3 shows five averaged (by spatial pixel and line) spectral measurements of the black body observed at temperatures of $20{ }^{\circ} \mathrm{C}, 30{ }^{\circ} \mathrm{C}, 40{ }^{\circ} \mathrm{C}, 60{ }^{\circ} \mathrm{C}$, and $80{ }^{\circ} \mathrm{C}$ alongside theoretical black body spectra plotted as dotted lines. All of the measurements roughly resemble the predicted black body curve, but are slightly noisy. Fig. 4 shows the rms of each of the five measurements at the different temperatures and so is an indication of the noise that can be expected in the processed data. This is dependent on both temperature and wavelength and has a similar spectral profile to the measured radiance. This cannot be attributed to thermal noise in the detector, since it is cooled to a temperature of $63.10 \mathrm{~K}$ for the $40{ }^{\circ} \mathrm{C}, 60{ }^{\circ} \mathrm{C}$, and $80{ }^{\circ} \mathrm{C}$ data acquired on day 2 and to a temperature of $63.00 \mathrm{~K}$ for the remainder of the measurements on day 1 . The additional $20^{\circ} \mathrm{C}$ data set is stabilized at $63.40 \mathrm{~K}$ and the $30{ }^{\circ} \mathrm{C}$ at $63.10 \mathrm{~K}$. Throughout all measurements, the detector temperature varies no more than $1 \mathrm{~K}$. This corresponds to a maximum of $5 \times 10^{-6} \mathrm{~W} /\left(\mathrm{m}^{2} \mathrm{sr} \mu \mathrm{m}\right)$ thermal noise at the longest wavelength. At $9 \mu \mathrm{m}$, this corresponds to only $2 \times 10^{-6} \mathrm{~W} /\left(\mathrm{m}^{2} \mathrm{sr} \mu \mathrm{m}\right)$. Therefore, the difference between

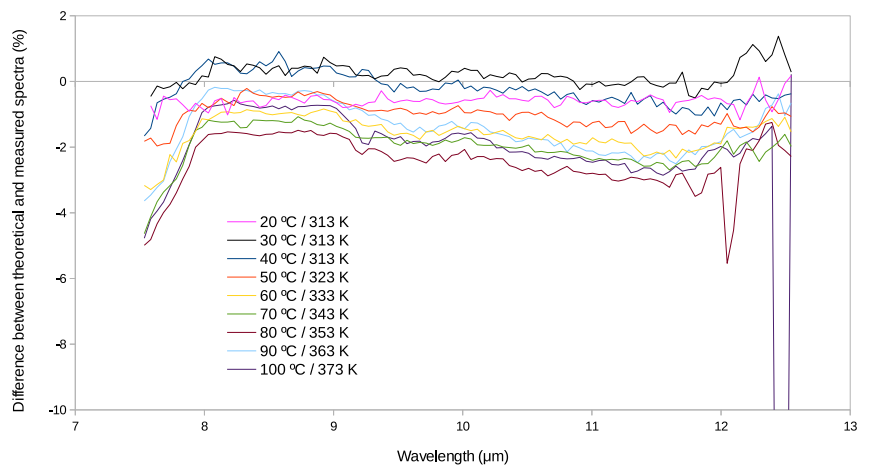

Fig. 5. Percentage difference between the averaged measured and theoretical spectra.

TABLE II

BLACK BODY TEMPERATURE MEASUREMENTS

\begin{tabular}{|l|c|c|c|}
\hline Black Body Set Temperature $^{\circ} \mathbf{C}$ & 40 & 60 & 80 \\
\hline FLIR measurement ${ }^{\circ} \mathbf{C}$ & 40.7 & 60.7 & 80.7 \\
\hline Temperature from fitting AisaOWL spectra $^{\circ} \mathbf{C}$ & 40 & 59.5 & 78.5 \\
\hline
\end{tabular}

the measured and theoretical spectra cannot be explained by thermal noise in the detector.

Comparing each of the spectra with the theoretical results, the measured temperature is within $2{ }^{\circ} \mathrm{C}$ of the set temperature. The results for each set temperature are shown in Table II. The temperature has also been measured with the FLIR camera for comparison.

The difference between the measured and predicted spectra does increase with temperature, though there is some variations in this trend, as can be seen in Fig. 5. For example, the $90{ }^{\circ} \mathrm{C}$ spectrum has a smaller magnitude of error than the $80{ }^{\circ} \mathrm{C}$ data across the entire spectrum. This may be due to the uncertainty in the temperature of the external black body, which is also limited in absolute temperature accuracy. In all cases except for the $30{ }^{\circ} \mathrm{C}$ and $40{ }^{\circ} \mathrm{C}$ temperatures, the temperature measured by the AisaOWL is lower than the theoretical response for the temperature that was set, suggesting that part of this error is due to the imperfect emissivity of the black body.

Each spectrum shows a sharp increase in error below $8 \mu \mathrm{m}$, which is suspected by Specim of being due to absorption from moisture in the air. In the $8-9 \mu \mathrm{m}$ region, the error is smallest, by $1 \%$ to $2 \%$ compared with the longer wavelengths. Above $9 \mu \mathrm{m}$, the observations deviate from the theoretical spectra, possibly indicating reduced sensitivity in the higher wavebands. This is more pronounced as temperature increases indicating decreasing detector performance with increasing temperature in the longer wavelengths. Thus, the optimal bands for determining absolute temperature are in the 8-9 $\mu \mathrm{m}$ region. This corresponds to the spectral peak for the $60{ }^{\circ} \mathrm{C}-100{ }^{\circ} \mathrm{C}$ curves, but not the $20{ }^{\circ} \mathrm{C}-50{ }^{\circ} \mathrm{C}$ spectra, so the spectral peak cannot be considered to be the region of best response.

\section{B. Warm-Up Time}

The manufacturer recommends a warm-up time of 15-30 min for the detector to cool and stabilize. Data collected before the recommended warm-up time of 15-30 min (cold 


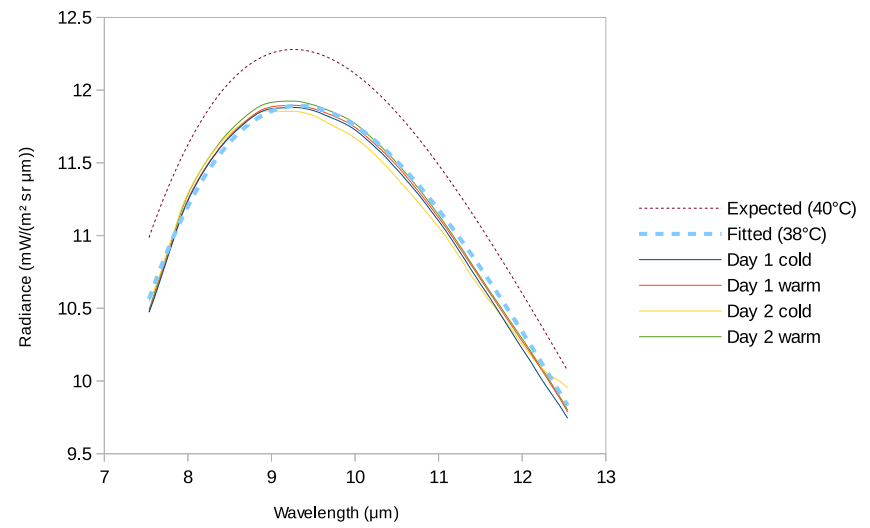

Fig. 6. Average spectra for each of the data sets alongside the predicted black body spectrum of $40{ }^{\circ} \mathrm{C}$ and the fitted black body spectrum of $38{ }^{\circ} \mathrm{C}$.

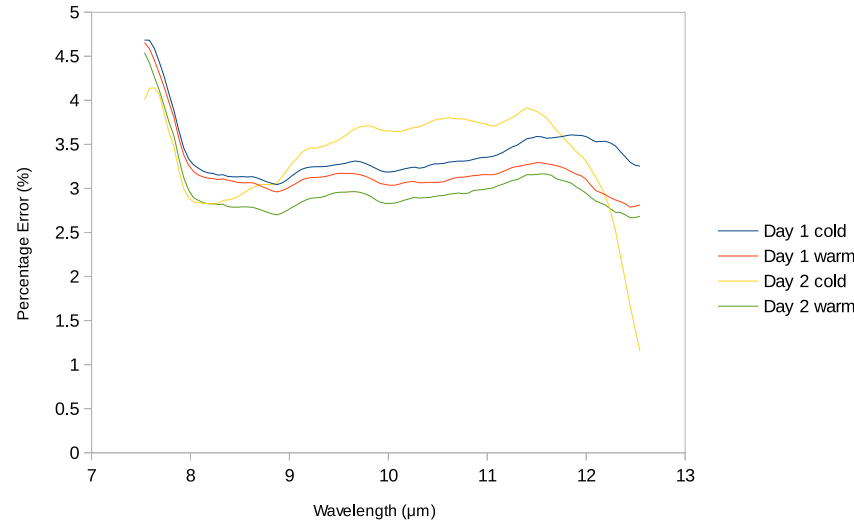

Fig. 7. Percentage difference between predicted and averaged measured spectra for each of the data sets.

data) have been compared with data collected after the warmup time that has elapsed (warm data). Fig. 6 shows the mean spectra (across all spatial pixels and lines) for each of the data sets alongside the predicted black body spectrum of $40^{\circ} \mathrm{C}$ and the closest fitting spectrum of $38{ }^{\circ} \mathrm{C}$. Each of the spectra is similar, but the day 2 cold data, which have the shortest warmup time of $7 \mathrm{~min}$, are slightly noisier. This suggests that the reduced warm-up time has had a detrimental impact on sensor performance. To highlight this decrease in performance, the difference between the theoretical black body curve and each of the data sets is plotted in Fig. 7.

From Fig. 7, it is clear that a warm-up time of $7 \mathrm{~min}$ is not sufficient, as the day 2 cold spectral error has a completely different shape to those of the other three data sets, which are very similar to each other. The error in the day 2 cold data increases from around 3\% to over 3.5\% compared with the day 2 warm data and varies across the spectrum. The $3 \%$ error in each spectrum is due to the $2{ }^{\circ} \mathrm{C}$ inaccuracy previously found in the measurements, part of which may be due to the imperfect emissivity of the black body. The deviation below $8 \mu \mathrm{m}$ is thought to be due to absorption by water in the atmosphere and the increasing error beyond $9.5 \mu \mathrm{m}$ is likely to be due to the slightly poorer performance of these spectral bands in general.

\section{Integration Time}

Spectra were examined to identify the effects of increasing integration time on sensor performance. Most of the sensor

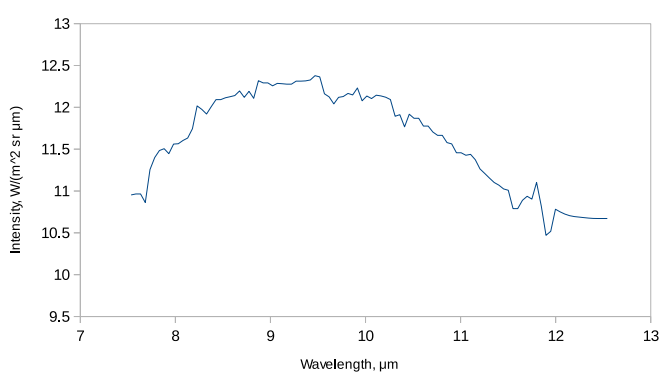

(a)

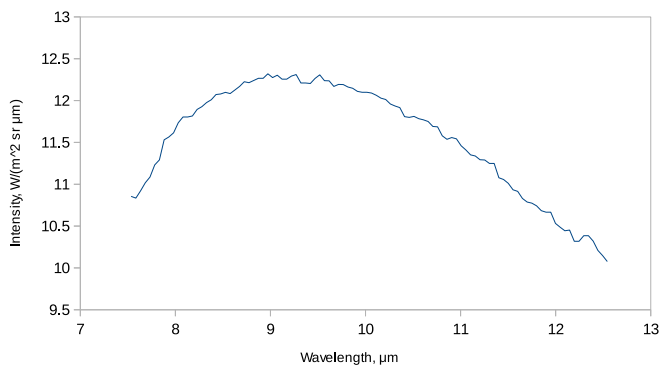

(b)

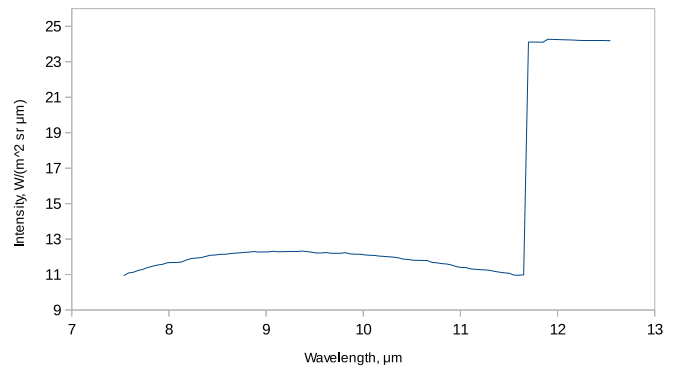

(c)

Fig. 8. Black body data acquired with three different integration times. (a) 0.18 , (b) 1.18 , and (c) $1.43 \mathrm{~ms}$

settings are independent, so integration time only has an impact on signal level. Nevertheless, too low signal can be compensated by spectral or spatial binning, but this reduces the spectral or spatial resolution, respectively. This option is used with other optical hyperspectral sensors, particularly in poor illumination, but has not been required for the AisaOWL.

Not all data could be processed with Specim's calibration tool, due to the high number of blinking pixels identified. As one expects, a very small integration time results in a noisy spectrum. This could obscure information when collecting data against nonblack body material. As the integration time increases, the spectra become less noisy until the optimum time of approximately $1.18 \mathrm{~ms}$. Beyond this integration time, the spectra develop an intensity peak in the longer wavelengths greater than $10.5 \mu \mathrm{m}$, possibly due to saturation in these bands. Example spectra for three different integration times, including the optimum time, are shown in Fig. 8.

Statistics for each data file have been calculated to determine whether there is a linear change in performance with integration time. Fig. 9 shows the rms error in the averaged spectrum for each integration time when compared with the predicted black body spectrum. The rms error generally decreases until the optimal integration time of $1.18 \mathrm{~ms}$, followed by a sharp 


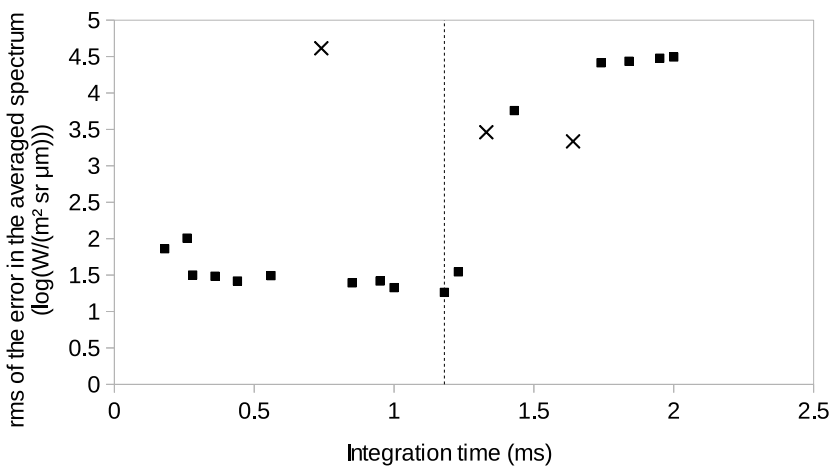

Fig. 9. RMS of the error in the averaged spectrum for each integration time. Crosses: data that do not have fresh black body calibration data. Vertical dashed line: manufacturer recommended optimal integration time of $1.18 \mathrm{~ms}$.

rise as integration times become longer. The decrease in performance is exponential rather than linear. The points indicated by crosses are measurements where internal black body collection failed, so were processed with the next closest calibration file and can be considered anomalous. These highlight that data must be calibrated with black body data acquired with the same integration times, since the calibration data are not normalized by time.

\section{Delayed Calibration}

Specim suggests that the black body data should be collected every $30 \mathrm{~min}$ as within this time, sensor performance should not significantly be affected. Data were reprocessed with black body files collected at different times to examine the effect on data quality of limited calibrations and an increase in time between black body calibration and actual data collection. The quality of the data processed using the various calibration files has been quantified by subtracting the measured spectra from the $30{ }^{\circ} \mathrm{C}$ theoretical black body spectrum.

Fig. 10 shows the averaged spectra from the $30{ }^{\circ} \mathrm{C}$ data set after processing with various calibration files acquired at increasing time delays. The spectra are labeled according to the delay in minutes from the no delay "T" spectrum, with negative delays indicating that the calibration black body data were acquired before data acquisition. There appears to be increasing error at the higher wavelengths for the longer delays, suggesting that these bands drift more. Thus, over larger time periods between black body and data collection, the quality of the calibrated data diminishes. Between 8 and $10 \mu \mathrm{m}$, the error stays within a percentage point indicating that this region will have better quality in processed data. The no delay " $\mathrm{T}$ " calibration file produces an error close to $0 \%$ with little variation across the spectrum. This indicates the best results, as expected with the shortest delay.

Fig. 11 shows the root-mean-square differences between the measured and predicted spectra for each calibration file used. A linear regression has been performed to calculate the trend for increasing error with time delay and is represented by the dotted lines on the graph. The R-squared value is 0.95 and the standard error is 0.42 . The "T-14" data point has been excluded from the linear regression as the error is in the opposite direction.

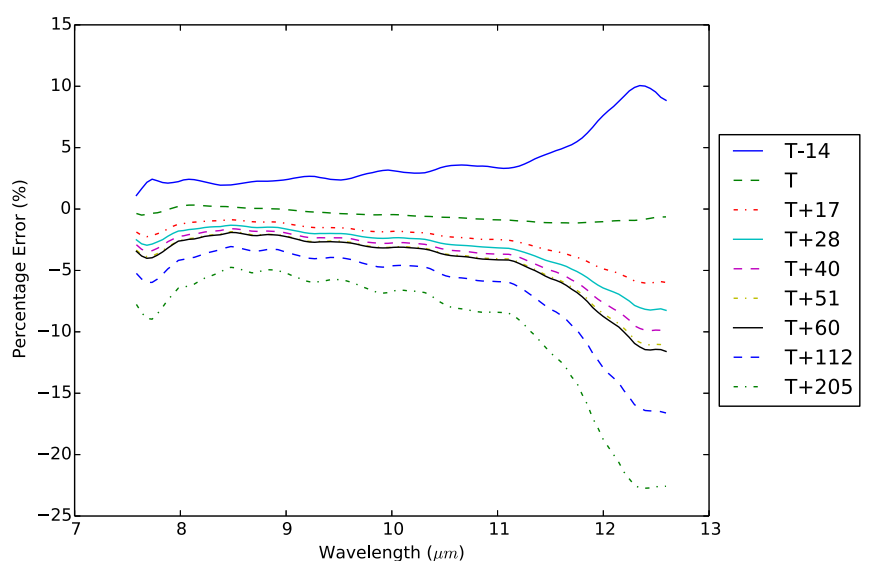

Fig. 10. Percentage difference between the predicted spectrum and the $30^{\circ} \mathrm{C}$ test data processed with calibration data taken at different times.

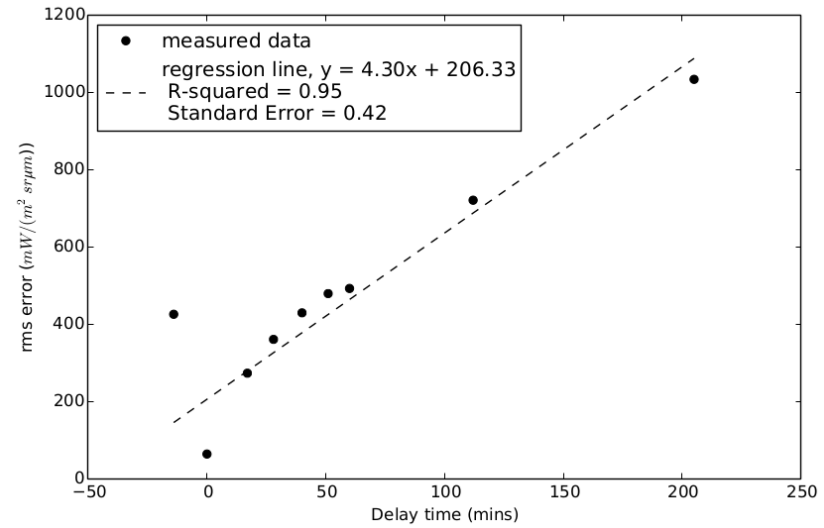

Fig. 11. RMS differences between the measured and predicted spectra, excluding the three anomalous spectra.

As well as the delay between data acquisition and internal black body acquisition, there are other factors determining the suitability of calibration data. The sensor settings, particularly integration time, must be identical, as the calibration data are not normalized by time. Also, an event has been observed, where every pixel undergoes a random jump in output level at the same time, which would render the calibration data unsuitable for part of that acquisition.

\section{CONClusion}

This paper has presented an independent characterization of the AisaOWL thermal hyperspectral instrument to develop understanding of its capability for environmental remote sensing. This analysis is essential for establishing high quality operational procedures and in giving confidence to users of the data. This characterization has focused on bench calibration tests to validate the temperature accuracy of spectral measurements and the recommended warm-up, integration, and calibration times. The characterization of blinking pixels is addressed in a separate study.

Measurements of a black body at different temperatures have provided a maximum absolute temperature error of $2{ }^{\circ} \mathrm{C}$. This error is not uniform across the spectrum and is minimal in the $8-9 \mu \mathrm{m}$ region. 
A sensor warm-up time of $15 \mathrm{~min}$ has proved sufficient. For warm-up times shorter than this, a small decrease in sensor performance has been observed, particularly in the bands beyond $9.5 \mu \mathrm{m}$. This is observed as an approximately $1 \%$ change in the percentage error in bands beyond $9.5 \mu \mathrm{m}$ compared with data collected after the 30-min warm-up time.

The experiments have validated Specim's advised integration time of $1.18 \mathrm{~ms}$ and have built on this to provide an optimal range of $0.85-1.2 \mathrm{~ms}$. Integration times outside of this region show that the performance of the sensor decreases on an exponential scale.

It has been observed in the data processed with different black body calibration data that the detector response changes over time. Usually this change in detector response is minor over the time of a few hours, resulting in an approximate 5\% change across the central bands. For bands below $8 \mu \mathrm{m}$ and above $11 \mu \mathrm{m}$, this change is much greater, approaching $10 \%$ below $8 \mu \mathrm{m}$ and exceeding $20 \%$ above $11 \mu \mathrm{m}$. This means that most flight data can be accurately calibrated at the beginning or end of the flight, but the outlying bands will be less reliable. However, the possibility for a rapid and dramatic change must be taken into account when selecting calibration data, and it is recommended to collect black body calibration data for each flight line. Best practice remains to collect the black body calibration data after each flight line acquisition, which can be set to occur manually or automatically. Typically 1024 frames are acquired from both of the black bodies, which are mounted in a unit below the instrument and in turn slide into the field of view. When the sensor is functioning correctly, this usually takes approximately $3 \mathrm{~min}$, but can take longer.

\section{ACKNOWLEDGMENT}

The authors would like to thank C. MacLellan for his assistance in instrument calibration. They would like to thank H. Holma of Specim for answering numerous questions about the AisaOWL instrument and radiometric calibration tool. They would also like to thank G. B. M. Pedersen and the Icelandic Research Fund for allowing the Iceland imagery to be presented.

All data used in this paper have been provided by the Natural Environment Research Council (NERC) U.K. and the NERC Airborne Research Facility.

\section{REFERENCES}

[1] F. A. Kruse, J. W. Boardman, and J. F. Huntington, "Comparison of airborne hyperspectral data and EO-1 Hyperion for mineral mapping," IEEE Trans. Geosci. Remote Sens., vol. 41, no. 6, pp. 1388-1400, Jun. 2003, doi: http://dx.doi.org/10.1109/TGRS.2003.812908

[2] S. Hancock et al., "Waveform lidar over vegetation: An evaluation of inversion methods for estimating return energy," Remote Sens. Environ., vol. 164, pp. 208-224, Jul. 2015, doi: http://dx.doi. org/10.1016/j.rse.2015.04.013

[3] R. Gaulton and T. J. Malthus, "LiDAR mapping of canopy gaps in continuous cover forests: A comparison of canopy height model and point cloud based techniques," Int. J. Remote Sens., vol. 31, no. 5, pp. 1193-1211, 2010, doi: http://dx.doi.org/10.1080/ 01431160903380565

[4] R. A. Hill, A. K. Wilson, M. George, and S. A. Hinsley, "Mapping tree species in temperate deciduous woodland using time-series multispectral data," Appl. Vegetation Sci., vol. 13, no. 1, pp. 86-99, 2010, doi: http://dx.doi.org/10.1111/j.1654-109X.2009.01053.x
[5] P. D. Hunter, A. N. Tyler, L. Carvalho, G. A. Codd, and S. C. Maberly, "Hyperspectral remote sensing of cyanobacterial pigments as indicators for cell populations and toxins in eutrophic lakes," Remote Sens. Environ., vol. 114, pp. 2705-2718, Nov. 2010, doi: http://dx.doi.org/10.1016/j.rse.2010.06.006

[6] G. B. M. Pedersen, "Semi-automatic classification of glaciovolcanic landforms: An object-based mapping approach based on geomorphometry," J. Volcanol. Geothermal Res., vol. 311, pp. 29-40, Feb. 2016, doi: http://dx.doi.org/10.1016/j.jvolgeores.2015.12.015

[7] D. A. Quattrochi and J. C. Luvall, "Thermal infrared remote sensing for analysis of landscape ecological processes: Methods and applications," Landscape Ecol., vol. 14, no. 6, pp. 577-598, 1999, doi: http://dx.doi.org/10.1023/A:1008168910634

[8] E. Ben-Dor, N. Goldlshleger, Y. Benyamini, M. Agassi, and D. G. Blumberg, "The spectral reflectance properties of soil structural crusts in the 1.2- to $2.5-\mu \mathrm{m}$ spectral region," Soil Sci. Soc. Amer. J., vol. 67, no. 1, pp. 289-299, 2001, doi: http://dx.doi.org/ $10.2136 /$ sssaj2003.2890

[9] B. Connor, I. Carrie, R. Craig, and J. Parsons, "Discriminative imaging using a LWIR polarimeter," Proc. SPIE, vol. 7113, p. 71130K, Oct. 2008, doi: http://dx.doi.org/10.1117/12.802176

[10] G. Notesco, Y. Ogen, and E. Ben-Dor, "Mineral classification of Makhtesh Ramon in Israel using hyperspectral longwave infrared (LWIR) remote-sensing data," Remote Sens., vol. 7, no. 9, pp. 12282-12296, Sep. 2015, doi: http://dx.doi.org/10.3390/rs70912282

[11] W. R. Johnson et al., "HyTES: Thermal imaging spectrometer development," in Proc. IEEE Aerosp. Conf., Mar. 2011, pp. 1-8, doi: 10.1109/AERO.2011.5747394.

[12] A. Gillespie, S. Rokugawa, T. Matsunaga, J. S. Cothern, S. Hook, and A. B. Kahle, "A temperature and emissivity separation algorithm for Advanced Spaceborne Thermal Emission and Reflection Radiometer (ASTER) images," IEEE Trans. Geosci. Remote Sens., vol. 36, no. 4, pp. 1113-1126, Jul. 1998, doi: http://dx.doi.org/10.1109/36.700995

[13] J. A. Morgan, "The definition of surface emissivity in thermal remote sensing," in Proc. IEEE Aerosp. Conf., vol. 5. Mar. 1998, pp. 159-169, doi: http://dx.doi.org/10.1109/AERO.1998.685813

[14] Z.-L. Li, F. Becker, M. P. Stoll, and Z. Wan, "Evaluation of six methods for extracting relative emissivity spectra from thermal infrared images," Remote Sens. Environ., vol. 69, no. 3, pp. 197-214, 1999, doi: http://dx.doi.org/10.1016/S0034-4257(99)00049-8

Laura Harris was born in Nuneaton, U.K., in 1986. She received the M.Sc. degree in chemistry and physics within the Natural Sciences Programme from Durham University, Durham, U.K., in 2008, and the M.Sc. degree in defence sensors and data fusion from Cranfield University, Cranfield, U.K., in 2013.

From 2008 to 2014, she was with the Defence Science and Technology Laboratory, Salisbury, U.K. She is currently with the Plymouth Marine Laboratory, Plymouth, U.K., focusing on airborne Earth observation.

Ms. Harris is a Chartered Member of the Institute of Physics.

Mark Warren received the Ph.D. degree in engineering surveying and space geodesy from the University of Nottingham, Nottingham, U.K., in 2007. His thesis work was on subsidence monitoring using satellite SAR data.

In 2008, he joined the Plymouth Marine Laboratory, Plymouth, U.K., where he is currently a Remote Sensing Scientist. His research interests include remote sensing, including airborne hyperspectral and lidar surveying, satellite ocean monitoring, and developing data processing algorithms.

Dr. Warren is a member of the Remote Sensing and Photogrammetry Society.

Michael Grant was born in U.K., in 1975. He received the B.S. and $\mathrm{Ph} . \mathrm{D}$. degrees in computer science from the University of Southampton, Southampton, U.K., in 1997 and 2002, respectively.

Since 2005, he has been with the Plymouth Marine Laboratory, Plymouth, U.K., initially as a Remote Sensing Scientist and then as a Senior Earth Observation Scientist. He is involved in the application of large scale computing to sensor observations, with particular interests in uncertainty estimation, calibration, and climate-grade reprocessing.

Gary M Llewellyn was born in Bristol, U.K., in 1966. He received the B.Sc. degree (Hons.) in environmental sciences and the Ph.D. degree from the University of Southampton, Southampton, U.K., in 1998 and 2009, respectively.

Since 2007, he has been with the Natural Environment Research Council, Swindon, U.K., with their airborne remote sensing capability, and promotes and coordinates airborne remote sensing for environmental science with specific regard to optical systems observing terrestrial vegetation, sampling and calibration, and validation of data. 